\title{
Involvement of dysregulated coding and long non-coding RNAs in the pathogenesis of strabismus
}

\author{
WEN-XIU MA* ${ }^{*}$ XIAO-GANG HUANG* ${ }^{*}$, TIAN-KE YANG ${ }^{*}$ and JING-YAN YAO \\ Department of Ophthalmology, The First Affiliated Hospital of Soochow University, Suzhou, Jiangsu 215000, P.R. China
}

Received November 12, 2017; Accepted March 9, 2018

DOI: $10.3892 / \mathrm{mmr} .2018 .8832$

\begin{abstract}
Strabismus is a common ocular disorder in children and may result in exterior abnormalities and impaired visual functions. However, the detailed pathogenesis of strabismus unclear. The present study assessed the comprehensive analyses on the roles of RNAs in the development of strabismus. The public datasets of strabismus and the corresponding control tissues were downloaded from the Gene Expression Omnibus (GEO). Reannotations of the dysregulated coding and long non-coding RNAs (lncRNAs) and functional enrichments of the differently expressed genes (DEGs) were conducted. A total of 790 DEGs were screened (648 upregulated and 142 downregulated) in the present study. Among the DEGs, a total of 32 differently expressed lncRNAs were detected (14 upregulated and 18 downregulated). When the Gene Ontology (GO) enrichment was considered, it was identified that a total of $143 \mathrm{GO}$ terms ( 82 for biological process, 31 for cellular component and 30 for molecular function) were identified. Among all the 57 detected Kyoto Encyclopedia of Genes and Genomes (KEGG) pathways, the phagosome pathway, which was labeled as hsa004145, demonstrated the most bioinformatics importance. However, most lncRNAs, except LINC01279 and LOC643733, indicated <3 target mRNAs and were not suitable for advanced bioinformatics analyses. Bioinformatics analyses demonstrated that there was a GO term for each IncRNA (proteinaceous extracellular for LINC01279 and cell surface for LOC643733). In conclusion, a set of coding RNA as well as lncRNAs differentially expressed in strabismus EOM samples were indicated. Notably, the present findings important information for advanced pathogenesis research and biomarkers detection.
\end{abstract}

Correspondence to: Dr Jing-Yan Yao, Department of Ophthalmology, The First Affiliated Hospital of Soochow University, 188 Shizi Road, Suzhou, Jiangsu 215000, P.R. China

E-mail: yjy00547@163.com

*Contributed equally

Key words: strabismus, extraocular muscles, microarray, long non-coding RNAs, bioinformatics

\section{Introduction}

Strabismus, which is an imbalance of both eyes, has been reported to be a common ocular disorder in childhood populations (1). Serious and persistent strabismus may lead to both exterior abnormality and impaired visual functions, including binocularity, stereo vision and visual activity. Uncorrected strabismus is also one of the most common causes of visual impairment and some cases were accompanied with amblyopia (2). Considering the infaust consequences of strabismus, early diagnosis and treatments were required. Nowadays, the treatments of strabismus included both surgical treatment and visual function training. Even operation could improve the strabismal status in most patients, the long-term prognosis of certain sub-type strabismus, including intermittent strabismus and AV signs, were quite unsatisfactory. Residual strabismus and impaired visual functions were present in some cases and would conduct an advanced surgical or rehabilitative interventions. Better understanding of the pathogenesis of strabismus would help in the improved diagnosis and treatments in the future.

According to a generally accepted theory, the incidence of strabismus was caused by the impairment of central neural pathways and maladjusted extraocular muscles (EOMs) would behave strabismal symptoms (3). EOM, which demonstrated crucial roles in the control of eye position, certainly played a key role in the development of strabismus. Initial opinion demonstrated that the EOMs in strabismus cases were not pathologically altered, however, this opinion was challenged by most recent studies. In a previous study based on immunofluorescence multiple-marker method, it was found that the number of Pax7-positive cells/satellite cells in anterior portion of EOMs was higher (4). Differently disputation of subtype cells would provide both understanding of the function of EOM and guide for the strabismus surgery. Our team also focused on the ultrastructure and pathological changes in patients with strabismus. In a study based on clinical EOM samples of intermittent exotropia, it was found that the significantly higher levels of myosin and actin was detected in adolescent group comparing with the adult group. When the ultrastructure was considered, electron microscopy was conducted to reveal sarcomere destruction, myofilament disintegration, collagen proliferation, and fibrosis between different age groups.

Considering that both molecular biomarkers and microstructure abnormality were detected in the EOMs in strabismus cases, it was quite important to detect the detailed 
pathogenesis of strabismus (5). Besides, it also demonstrated significant potential importance in the detection of molecular and structural biomarkers in the diagnosis, classification and prognosis of strabismus management. A study by Altick et al was conducted to detect the gene expression profile in the EOMs from strabismal cases and normal controls (6). A total of 604 genes in strabismal EOM samples were detected based on the microarray analysis and advanced PCR array identified the significant muscle-specific genes expression pattern. However, all the previous studies focused on the coding RNA expression pattern. Noncoding RNAs, especially long non-coding RNAs (lncRNAs) which was the noncoding RNA transcripts of above 200 nucleotides that do not encode proteins, were also reported to play key roles in different biological progresses. As showed in previous studies, lncRNAs were reported to be involved in cancer occurrence, organ development and homeostasis maintenance (7-9). As showed in previous studies, lncRNAs were also involved in ocular disorders, such as diabetic retinopathy, choroidal neovascularization and age-related macular degeneration (10-12). Considering that lncRNAs could also regulate the function maintenance of muscles, it was quite important and interesting to detect the pathogenic roles of IncRNAs in the development of strabismus. As there was high-throughput data available in public databases, the re-annotations and data mining would provide us updated knowledge on the development of strabismus. The aim of this study to determine the expression pattern of both coding and lncRNAs in the EOM samples from strabismus cases and thus provide new understanding in the pathogenesis of strabismus with public data. Comprehensive analyses and updated knowledge on the roles of RNAs in the development of strabismus would provide potential clues for the detection of diagnostic, therapeutic and prognostic targets.

\section{Materials and methods}

Microarray data. Gene expression profiles of four strabismic and four normal EOM samples were downloaded from the Gene Expression Omnibus (GEO) database (http://www.ncbi.nlm.nih.gov/geo/). All the strabismic samples were from independent samples, while one of the controls were repeated samples of the other three samples. There were 3 females and 1 male in the strabismus group while 2 females and 2 males in the control group. No significant difference was detected in the age distribution between the case $(22.5 \pm 27.11)$ and control $(16.75 \pm 15.09)$ group $(\mathrm{P}=0.4493)$. The deviation angles of four strabismic cases were approximately $12^{\circ}-14^{\circ}$, $30^{\circ}, 45^{\circ}$ and approximately $45^{\circ}-55^{\circ}$. All the microarray analyses were conducted using Affymetrix Human Genome U133 Plus 2.0 Array (Affymetrix; Thermo Fisher Scientific, Inc., Waltham, MA, USA).

Data preprocessing and IncRNAs re-annotations. Expression data of both cases and controls in cel document were downloaded from GEO database with a serial accession number GSE38780 and used for advanced analyses. Considering that the dataset were based on the GPL570, the gene symbol as well as annotation information (refGene) were added into the downloaded datasets. Differently expressed genes (DEGs) between strabismic and control samples were identified using the LIMMA package (Linear Models for Microarray Data) in $\mathrm{R}$ software. To detect the DEGs, the adjusted P-value $<0.01$ and $\log \mathrm{FCl}>1$ cutoff criterion were obtained in the screening. All the DEGs were presented in heat map and volcano graph. Among all the DEGs, the refGene (NM, mRNA. NR, ncRNA. $\mathrm{XM}$, predicted mRNA model. XR, predicted ncRNA model) was used in the annotation of differently expressed lncRNAs.

Bioinformatics analyses and functional enrichment. To conduct the bioinformatics analyses based on the detected DEGs, the Gene Ontology (GO) functional enrichment based on Database for Annotation, Visualization and Integrated Discovery (DAVID) (13) and Kyoto Encyclopedia of Genes and Genomes (KEGG) pathway analyses (14) were both obtained for the functional analyses. GO analyses could be divided into three independent parts, including molecular function, biological process and cellular component. The detected protein or gene can match the corresponding GO number and then GO term would demonstrate the functional category or the cell location. KEGG is a database that integrates functional information about genomes, biochemical and organismal system. Application of KEGG pathway database would help in the comprehensive inferences for pathway mapping of DEGs. In this study, KOBAS 3.0 was used to carry on the KEGG pathway enrichment analysis and $\mathrm{P}<0.01$ was set as the screening condition.

Protein-protein interactions. Protein-protein interactions analyses were conducted using an a online bioinformatics tool, string (15) and a graphical presentation of the interaction network. Both the analyses were conducted by the Cytoscape 3.5.1 software (16). The functional node points of the interacting proteins were analyzed in advance. A total of eight evidence points that demonstrate the relationship between different nodes were obtained in the analyses and the associations with a combined_score $>0.9$ were listed in the network association list. Besides, the hub nodes, which demonstrated most significant potential reputational function, in this study were also detected.

IncRNA-mRNA co-expression network and functional enrichment of lncRNAs. For the moment, there was no available functional enrichment tool for high-throughput lncRNAs data. In general, functional related genes may demonstrate similar expression profiles and related expression pattern of lncRNAs-co-expressed mRNA would provide clues for the functional enrichments of lncRNAs. Thus it was an optional method for the functional enrichment of lncRNA by analyzing co-expressed mRNAs. In this study, WGCNA was used in the construction of IncRNA-mRNA co-expression network (17) and adjacency threshold was set at 0.85. Cytoscape software was obtained in the network formation as well. For advanced functional enrichments, both GO and KEGG pathway analyses of lncRNA-co-expressed mRNA were conducted in this study.

\section{Results}

DEGs and dysregulated lncRNAs. To detect the DEGs in the strabismic cases, the microarray data of 4 cases and 4 controls were used for advanced analyses. Using LIMMA with a P-value $<0.01$ and $\mid \log \mathrm{FCl}>1.0$, a total of 790 DEGs 


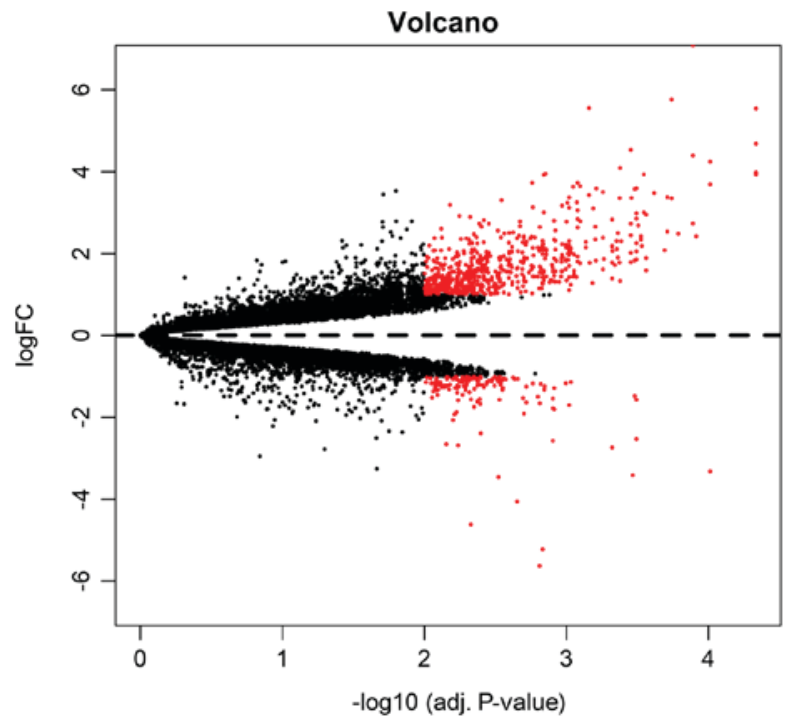

Figure 1. The volcano plot of the differently expressed genes. The $\mathrm{x}$ axis is $-\log 10^{\text {(P-value) }}$ while the y axis is $\log \mathrm{FC}$. Black dots idnicated non-differently expressed genes while the red dots indicated the differently expressed genes.

were screened (648 upregulated and 142 downregulated). Both volcano plot (Fig. 1) and clustering heatmap (Fig. 2) were used in the presentation of the DEGs. In the volcano plot, the DEGs were marked red among all the detected genes. In another way, clustering heatmap provided a graphical review of DEGs with upregulated (marked red) and downregulated (marked green) genes. To detect the most dysregulated genes of significance, we presented the top 10 DEGs in Table I. Among the 10 DEGs (TNMD, HBB, FNDC1, PTHLH, CRISPLD1, NPTX2, COL1A2, CHRDL1, CYS1 and SFRP2), only NPTX2 demonstrated a downregulation pattern.

Considering the important reputational role of IncRNAs in different biological progress, we also conducted advanced analyses to detect differently expressed lncRNAs. In this study, a total of 32 differently expressed lncRNAs were detected. Among all the detected lncRNAs, 14 lncRNAs were upregulated and the rest 18 were downregulated. The detected lncRNAs were presented in Fig. 3.

GO enrichment analysis. To further detect the roles of DEGs in the pathogenesis of strabismus, GO enrichment analysis was conducted in this study. By dividing all the related GO terms in to different progresses, it was found that a total of $143 \mathrm{GO}$ terms were identified in this study. Among all the detected terms, there were 82 in biological process, 31 in cellular component and 30 in molecular function. The detailed constituent of all the GO terms of significance were displayed in Fig. 4 and the top 10 most important terms were listed in Table II.

KEGG pathway analysis. KEGG enrichment analysis was also conducted to map the DEGs into regulation pathways. With the analyses by KOBAS 3.0 and $\mathrm{P}<0.01$ as screening criteria, a total of 57 evaluated pathways demonstrated statistical significance. Among all the evaluated pathways, phagosome, pathogenic Escherichia coli infection, ECM-receptor interaction, PI3K-Akt signaling pathway, regulation of actin cytoskeleton, focal adhesion, cell adhesion molecules, malaria,

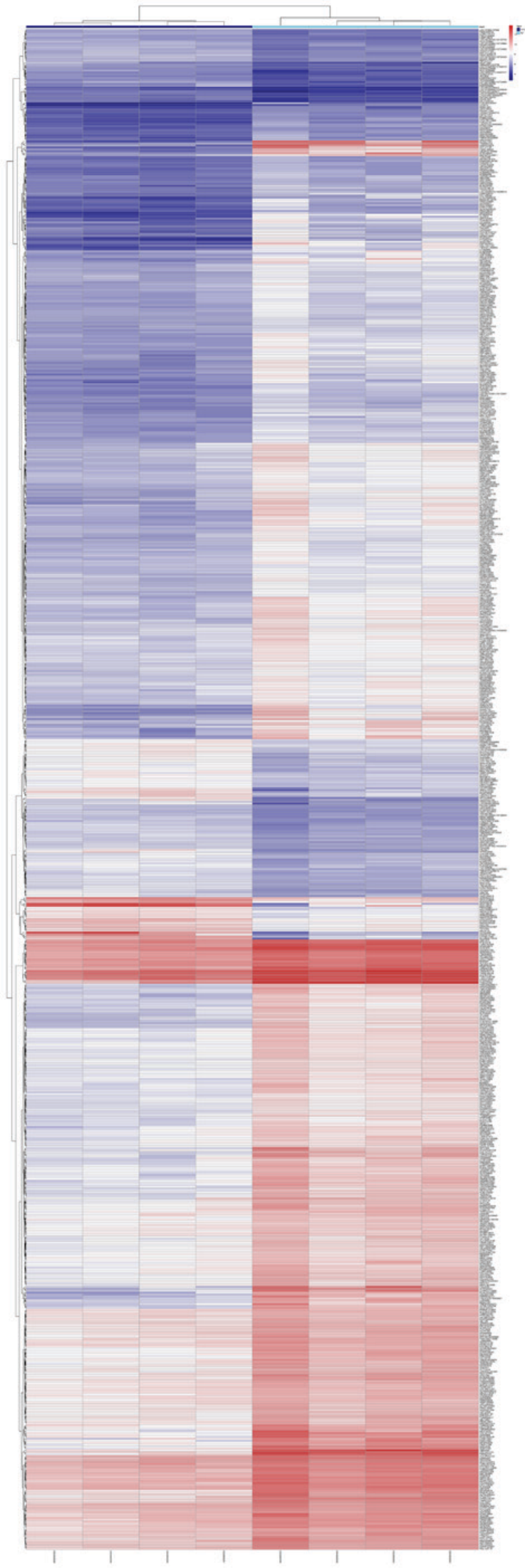

Figure 2. Heatmap of all differently expressed genes. The abscissa is for the sample serial number and the ordinate is for the differently expressed gene. Red blocks indicated upregulation and the blue blocks indicated downregulation. 
Table I. Differently expressed genes between strabismic cases and normal controls.

\begin{tabular}{lcccccc}
\hline Gene & Id & $\begin{array}{c}\text { Log value of } \\
\text { fold change }\end{array}$ & $\begin{array}{c}\text { Average } \\
\text { expression value }\end{array}$ & $\mathrm{t}$ & $\begin{array}{c}\text { Adjusted } \\
\text { P-value }\end{array}$ \\
\hline TNMD & 64,102 & 5.539642628 & 8.654139143 & 18.94555632 & $2.53 \times 10^{-09}$ & $4.61 \times 10^{-05}$ \\
HBB & 3,043 & 3.924910079 & 10.86418424 & 17.3483421 & $6.10 \times 10^{-09}$ & $4.61 \times 10^{-05}$ \\
FNDC1 & 84,624 & 4.682337268 & 7.527809329 & 17.00657939 & $7.44 \times 10^{-09}$ & $4.61 \times 10^{-05}$ \\
PTHLH & 5,744 & 3.976736723 & 6.135764953 & 16.68278379 & $9.01 \times 10^{-09}$ & $4.61 \times 10^{-05}$ \\
CRISPLD1 & 83,690 & 4.244618812 & 7.381617504 & 15.04795712 & $2.50 \times 10^{-00}$ & $9.72 \times 10^{-05}$ \\
NPTX2 & 4,885 & -3.319432337 & 7.137493269 & -14.61908535 & $3.32 \times 10^{-00}$ & $9.72 \times 10^{-05}$ \\
COL1A2 & 1,278 & 3.687990231 & 10.9966917 & 14.617825 & $3.33 \times 10^{-00}$ & $9.72 \times 10^{-05}$ \\
CHRDL1 & 91,851 & 2.416155203 & 10.50052948 & 14.09099772 & $4.77 \times 10^{-08}$ & 0.000121962 \\
CYS1 & 192,668 & 2.734788648 & 7.593811022 & 13.67185162 & $6.41 \times 10^{-08}$ & 0.000128488 \\
SFRP2 & 6,423 & 4.393543094 & 9.280684423 & 13.63393902 & $6.58 \times 10^{-08}$ & 0.000128488 \\
\end{tabular}

Gene for gene symbol and Id for gene ID.

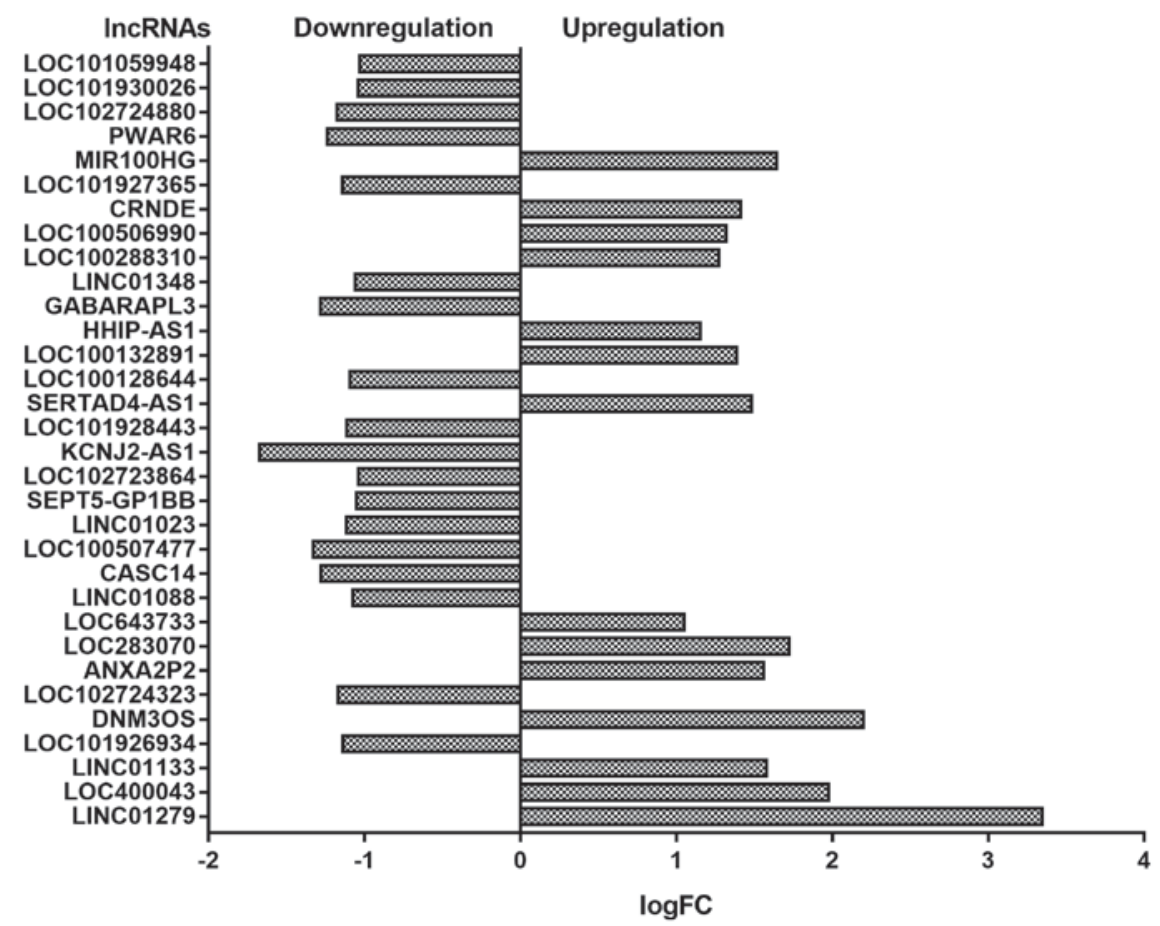

Figure 3. Differently expressed lncRNAs were displayed in the histogram. The $\mathrm{x}$ axis is logFC while the y axis is the names of lncRNAs.lncRNA, long non-coding RNA.

complement and coagulation cascades and TGF- $\beta$ signaling pathway were the top 10 significantly important pathways (Fig. 5A). While the phagosome pathway, which was labeled as hsa004145, demonstrated the most bioinformatics importance and may be related with the development of strabismus. The detailed pathway information of hsa004145 was presented in Fig. 5B.

Protein-protein interactions and function module analysis. Protein interactions analysis between DEGs were would provide the function enrichment as well as detect the hot point of significance. In this study, the detailed PPI network was showed in Fig. 6A. In the function module analysis, it was found that PPBP, COL3A1, GNG12, COL1A2 and AGT were reported to be the hub mode of the PPI network and the detailed interaction modes were presented in Fig. 6B.

lncRNA-mRNA co-expression network and functional analyses. Through WGCNA software, the top 10 most significant IncRNA-mRNA co-expression relationships detected and presented in Fig. 7. Among all the detected co-expression relationships, the related mRNAs were selected for advanced functional analyses. Most lncRNAs, except LINC01279 and LOC643733, indicated less than 3 target mRNAs and were not suitable for advanced bioinformatics analyses. Through advanced GO enrichment and KEGG pathway analysis, it was 
Table II. The top 10 identified Gene ontology terms of all the DEGs.

\begin{tabular}{lcr}
\hline Terms & Count & P-value \\
\hline GO:0070062 extracellular exosome & 220 & $2.99 \times 10^{-26}$ \\
GO:0005615 extracellular space & 136 & $3.14 \times 10^{-25}$ \\
GO:0005578 proteinaceous extracellular matrix & 54 & $5.07 \times 10^{-23}$ \\
GO:0005576 extracellular region & 142 & $8.77 \times 10^{-21}$ \\
GO:0031012 extracellular matrix & 53 & $3.43 \times 10^{-20}$ \\
GO:0030198 extracellular matrix organization & 42 & $4.77 \times 10^{-19}$ \\
GO:0007155 cell adhesion & 58 & $7.67 \times 10^{-15}$ \\
GO:0030199 collagen fibril organization & 17 & $3.92 \times 10^{-13}$ \\
GO:0005581 collagen trimer & 24 & $6.19 \times 10^{-13}$ \\
GO:0009986 cell surface & 57 & $2.95 \times 10^{-11}$
\end{tabular}

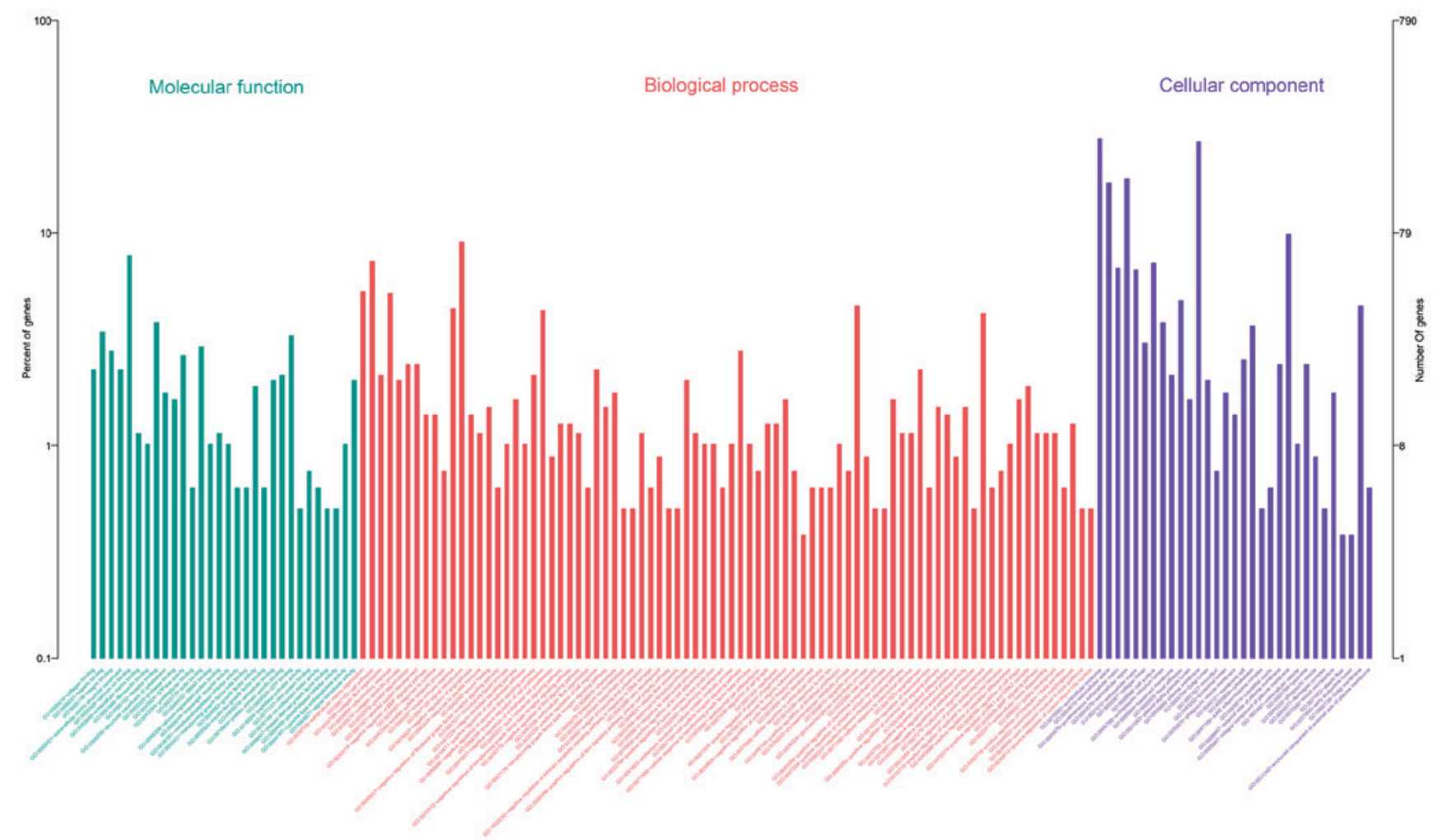

Figure 4. GO enrichment analysis of all the differently expressed genes. The abscissa is for the GO terms and the ordinate is for percent of genes. Cyan indicated molecular function terms, red indicated the biological process terms and blue indicated the cellular component terms.

found that no potential KEGG pathways could be detected in the related DEGs. However, there was a GO term for each lncRNA (proteinaceous extracellular for LINC01279 and cell surface for LOC643733) and they were displayed in Table III.

\section{Discussion}

The detailed mechanism for the development of strabismus was quite poorly understood. Even certain work has been conducted, the molecular pathogenesis of strabismus was litter detected in previous studies (18). In the present study, we re-analyzed the public microarray data and detected the effects of both coding and non-coding RNAs in the development of strabismus. By analyzing the RNA expression pattern of four strabismal and their paired controls, it was found that both coding and long non-coding RNAs were differently expressed in the EOM of strabismal patients. Advanced bioinformatics analyses provided updated evidence and clue in the understanding of strabismus.

Even different causes, including genetic, nerve regulated and structural modification, were reported to be associated with the development of strabismus (19-21), the EOM dysregulation was regarded as the most direct effector organ among various causes. Through scientific and systematic mRNA detection, the results would provide abundant knowledge on the incidence of strabismus. In 2012, Altick et al and colleagues conducted a microarray analyses based four pairs of strabismus cases and controls (6). It was found that a total of 604 genes were 

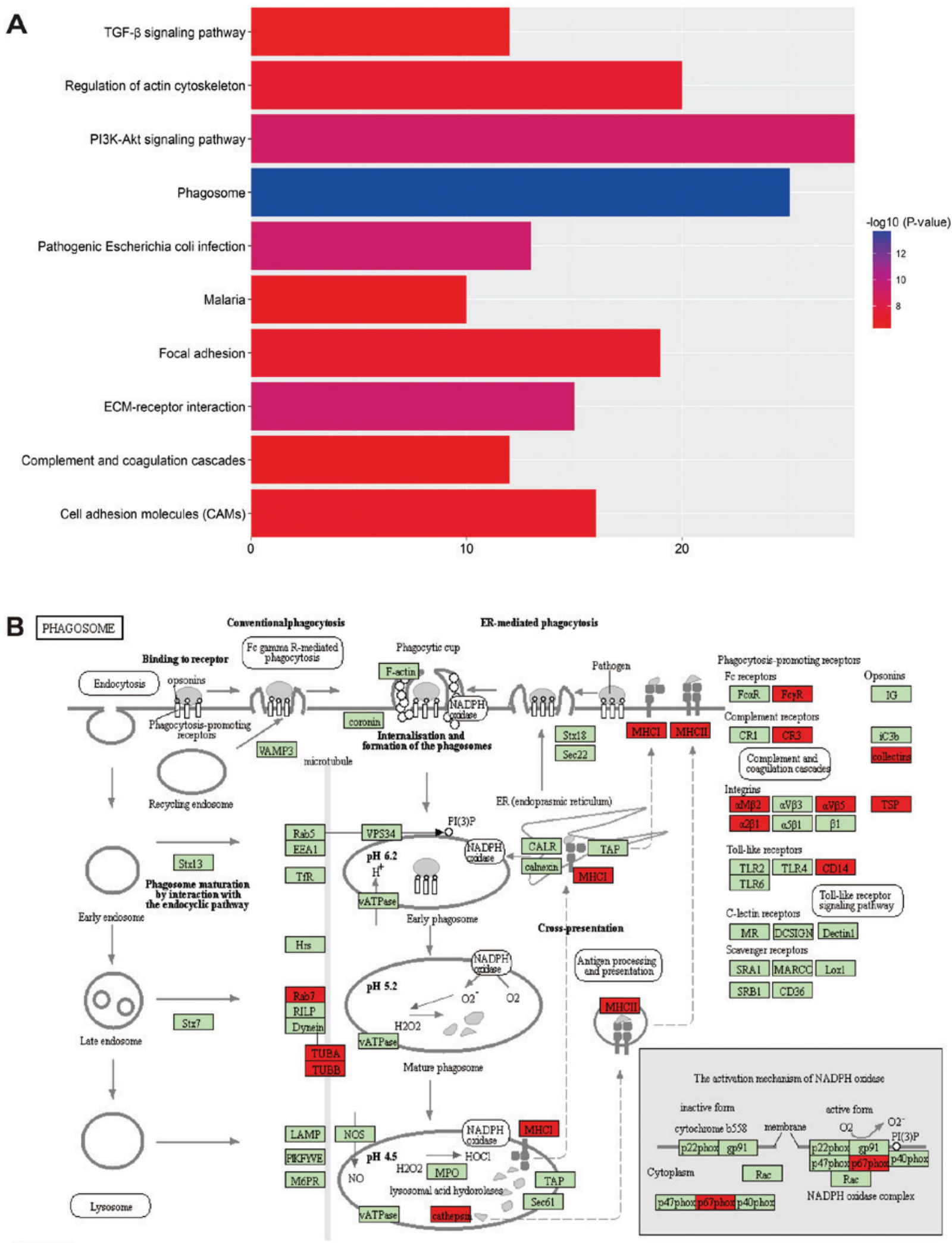

041453242417

Kanelica Laboratonis

Phagolysosome

Figure 5. KEGG enrichment analysis of all the differently expressed genes. (A) Top ten most significant KEGG pathways. (B) The phagosome KEGG pathway. The red blocks indicated the upregulaed genes and green blocks indicated the downregulaed genes. KEGG, Kyoto Encyclopedia of Genes and Genomes.

differently expressed in strabismic EOMs and advanced functional analyses demonstrated that DEGs involved in extracellular matrix structure (upregulated) and contractility (downregulated) demonstrated the most important effect. In our study, we modified the screening condition to adjusted $\mathrm{P}<0.01$ and $|\log \mathrm{FC}|>1$. Through adjustments of both P-value and $\log \mathrm{FCl}$, more DEGs with less variance and statistically significance would be detected in this study. In our study, more 
Table III. Gene Ontology terms of the lncRNAs.

\begin{tabular}{|c|c|c|c|c|c|}
\hline lncRNAs & Category & Term & Count & P-value & Genes \\
\hline LINC01279 & Cellular component & $\begin{array}{l}\text { GO:0005578 proteinaceous } \\
\text { extracellular matrix }\end{array}$ & 2 & 0.057543258 & CTHRC1, FBLN2 \\
\hline LOC643733 & Cellular component & GO:0009986 cell surface & 2 & 0.058599058 & TIMP2, ANXA4 \\
\hline
\end{tabular}

lncRNAs, long non-codingRNAs.

A

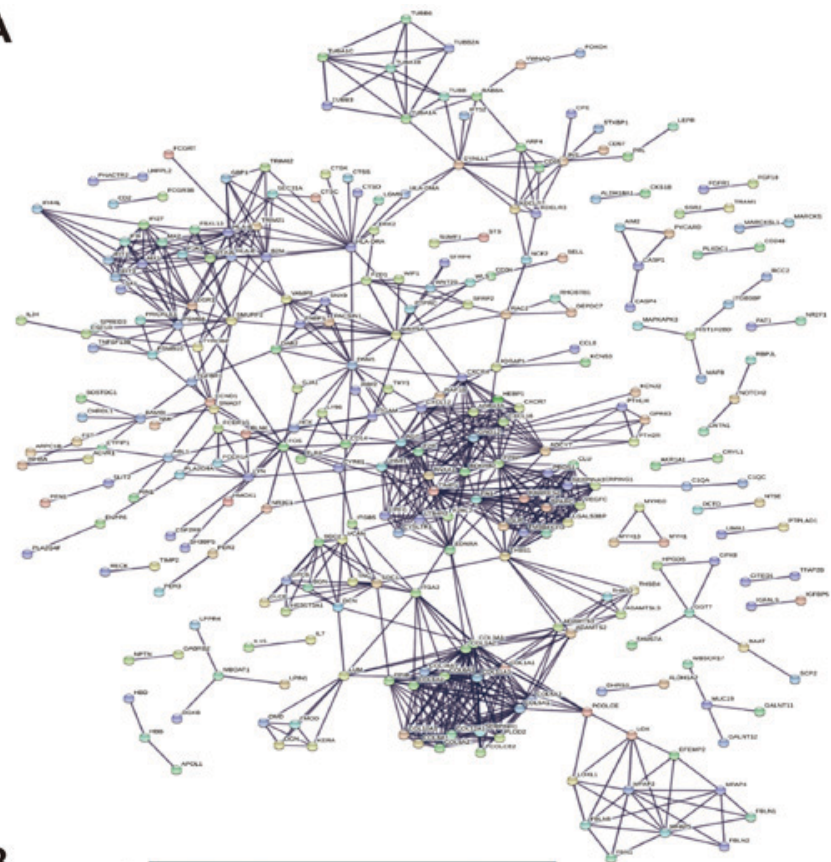

B

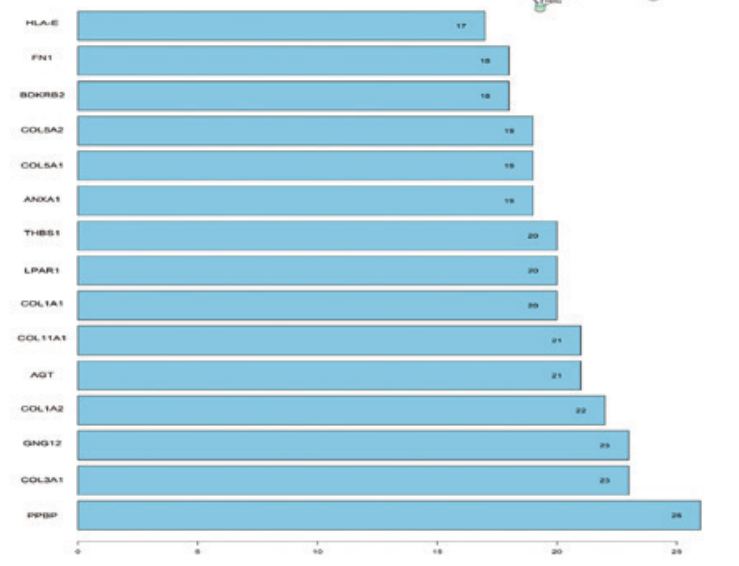

Figure 6. The protein-protein-interactions (PPI) analysis for all the differently expressed genes. (A) The PPI network of the detected genes. Circles represent genes, and lines represent interactions between genes, and the results within the circle represent the structure of proteins. The thrum color represents different evidence of the interaction between the proteins. A red line indicates the presence of fusion evidence; a green line-neighborhood evidence; a blue line-coocurrence evidence; a purple line-experimental evidence; a yellow line-text mining evidence; a light blue line-database evidence; a black line-coexpression evidence. (B) The numbers of each interacted genes. The $\mathrm{X}$ axis is numbers of genes while the $\mathrm{Y}$ axis is the names of genes.

DEGs were detected comparing with the primary analyses (790 DEGs vs. 604 DEGs). Based on the updated DEG data and GO analyses tool, it was reported that extracellular exosome, extracellular space and proteinaceous extracellular matrix were

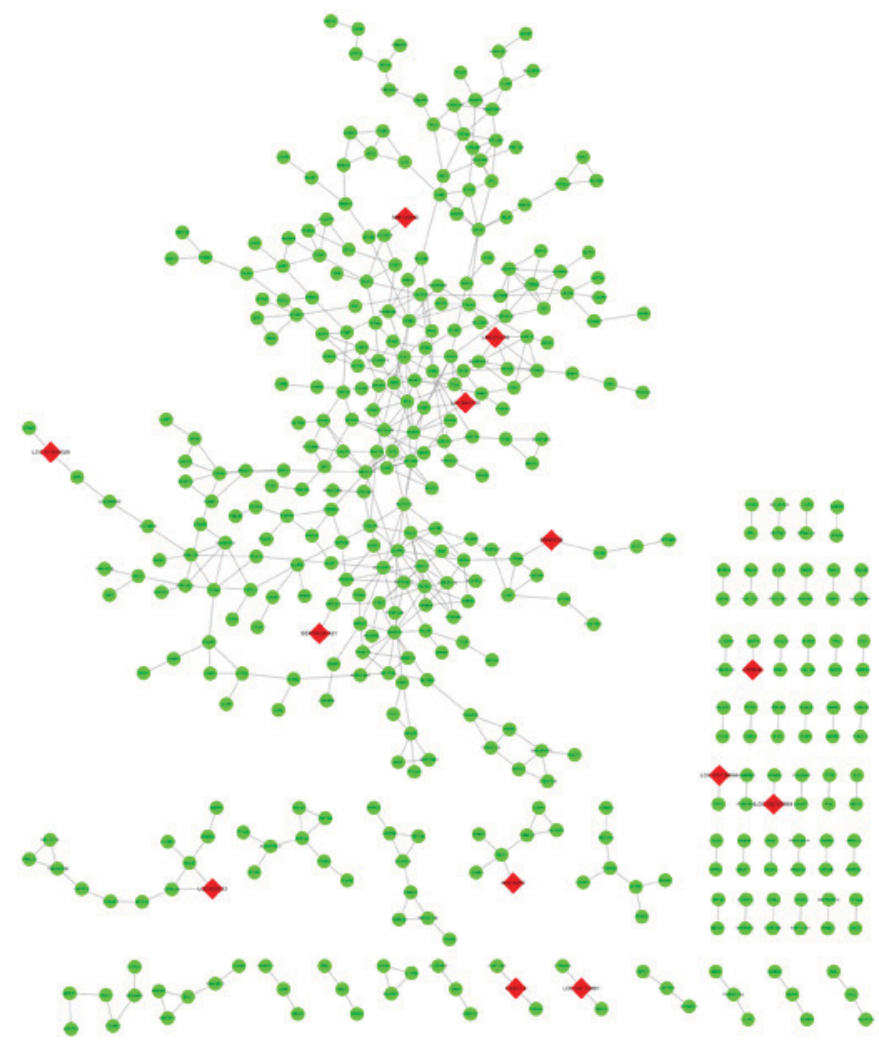

Figure 7. Co-expression network of all the lncRNA and mRNAs. The red rhombus indicated IncRNAs and the green circle indicated mRNAs. The line between the rhombus and circles indicated the co-expression relationship between the IncRNAs and mRNAs. IncRNA, long non-coding RNA.

the top three GO terms of significance. The previous detected pathways (extracellular matrix structure and contractility) were also significant in the updated analyses. It was also found that both conclusions indicated that the most important pathogenesis of strabismus was the modification of extracellular structure. It was quite easy to understand this conclusion demonstrating that the pathological study of strabismus demonstrated noticeable extracellular structure modification, including increased content of collagen fiber, and the gap between fibrous tissue, adipose tissue and muscle fiber widened (22). This phenomenon was also proved by a recent study in different study design. As reported by Agarwal et al, the difference in protein and RNA level of EOM samples from strabismic cases were detected proteomics, standard and customized PCR arrays, and microarrays (23). It was also reported that expression of collagens and regulators of collagen synthesis and degradation was upregulated in both RNA and protein level. These findings suggest that more work 
should be focused on the extracellular matrix modification in the development of strabismus.

KEGG pathway and PPI analyses were effective tools in the detection of key pathways and core regulators. By analyzing the information of updated DEGs, there was several interesting findings. TGF- $\beta$ signaling pathway, which was one of the top 10 related pathways, may demonstrate certain effects in the formation of strabismus. TGF- $\beta$, which is one of the most important growth factors in the pathogenesis of fibrotic diseases, demonstrated important role in the fibration formation and extracellular matrix modification (24). In the ocular disorders, TGF- $\beta$ was also reported to produce important regulative effect in corneal scarring, conjunctiva fibrosis, fibrosis of the lens capsule, strabismus development and proliferative vitreoretinopathy (25). Remarkable TGF- $\beta 1$ expression was observed in areas with excessive collagen deposition in the post-operative adhesion after strabismus surgery in experimental rabbit model and it indicated the effect of TGF- $\beta$ on the effects of postoperative recovery in strabismus surgery (26). Considering that local application of agents, including insulin-like growth factor-1 (IGF-1) and botulinum toxin $(27,28)$, for the treatment of strabismus demonstrated remarkable improvements, it was an interesting and potential important aspect in the application of anti-TGF- $\beta$ in the treatment of strabismus itself. It was phagosome pathway that demonstrate the most significant importance in this study, however, no previous study focused on this point. Phagosome, which was defined as the regulated uptake of large particles into cytosolic, membranebound vacuoles, demonstrated immunoregulatory for long (29). Nowadays, it has been reported that phagosome would provide important role in the organ development, homeostasis maintenance of inner environment and infection responses $(30,31)$. As autophagy was regarded as an important regulative progress in age related disorders, phagosomes pathway was reported to be involved in the development of age-related macular degeneration (32). Strabismus, which was a neurodevelopmental disease, may be regulated the regulation of phagosomes pathway and more work was required to be conducted in the future. However, it should be noticed that the bioinformatics analyses were based on the analyses of co-expressed mRNAs of the related lncRNAs. As we know, the IncRNAs usually played a role through DNA, RNA, protein and miRNAs, however no available bioinformatics tool could be used to demonstrate the annotations of the differently expressed lncRNAs. The co-expressed mRNAs with lncRNAs could only explain a part of the function of related lncRNAs and thus the bioinformatics analyses were just part of the global function of IncRNAs. The conclusion of this part should be considered with cautions.

Apart from coding RNAs, we also pay attention on the effect of lncRNAs on the incidence of strabismus in this study. As lncRNAs were reported to be involved in kinds of diseases, including cancer, cardiovascular disorders, diabetes and immune disorders (33-35). It was also reported that lncRNAs would demonstrate certain effects in the ocular disorders (36-38) and may produce potential therapeutic effects. However, no previous study focused the effect of lncRNAs in the development of strabismus by now. Considering EOM demonstrated direct pathogenic modification and provided primary therapy, the study on the effect of IncRNAs in the EOMs may provide important knowledge in this field. Previous studies demonstrated the effect of lncRNAs in the muscle function maintenance. A previous study based on in vivo and in vitro studies showed that a lncRNA, LncMyoD, demonstared regulative effects in skeletal muscle differentiation through blocking the translation of mRNA (39). Another study showed that s a novel lncRNA lnc133b, could regulate bovine skeletal muscle satellite cell, which was significantly actived in strabismus (40), proliferation and differentiation by mediating miR-133b (41). This study provide a potential thread in the research of lncRNAs on the pathopoiesis of strabismus. As mentioned in the above, TGF- $\beta$ was one of the most important regulator of strabismus development and treatment, a recent study by Tang et al indicated the detailed mechanism through which the lncRNA GAS5 regulated TGF- $\beta$-induced smooth muscle cell differentiation (42). The cross-talk between important lncRNAs and mRNA provided us abundant in the study of lncRNAs in the development of strabismus. Thus we conducted a relevant research on the expression of lncRNAs in the EOM samples of strabismus cases. Even many differently expressed lncRNAs were detected, however, no previous available literature demonstrated potential relation between lncRNAs and strabismus. Besides, the function enrichment analyses of lncRNAs was quite hard to conduct by now. In this study, we chose to conduct the functional analyses by analyzing the function of IncRNA-co-expressed mRNA. No satisfactory outcome was gained in this study. Besides, there was potential bias in this strategy because IncRNAs may demonstrate the effect through interaction with DNA or protein. Further in vitro and in vivo study as well as advanced bioinformatics analyses would provide better understanding of the effect of lncRNAs in the strabismus.

These results in this study demonstrated both coding and IncRNA produced certain effects in the development of strabismus. Functional enrichment analyses provide updated knowledge on the understanding of this disorder and thus provide potential therapeutic methods. However, the evidence of lncRNAs affecting the development should be proved in advanced studies. Further studies will be needed to conclusively demonstrate and elucidate the precise role of lncRNAs in strabismus.

\section{Acknowledgements}

Not applicable.

\section{Funding}

No funding was received.

\section{Availability of data and materials}

The datasets used and/or analyzed during the current study are available from the corresponding author on reasonable request.

\section{Authors' contributions}

WXM and JYY conceived and designed the experiments; WXM, TKY and JYY performed the experiments; WXM and XGH analyzed the data; WXM, XGH and JYY contributed reagents/materials/analysis tools and WXM and JYY wrote the manuscript. 


\section{Ethics approval and consent to participate}

Not applicable.

\section{Consent for publication}

Not applicable.

\section{Competing interests}

The authors declare that they have no competing interests.

\section{References}

1. Lambert SR: Population-based incidence of strabismus: Why is it important? JAMA Ophthalmol 135: 1053-1054, 2017.

2. Shapira Y, Machluf Y, Mimouni M, Chaiter Y and Mezer E: Amblyopia and strabismus: Trends in prevalence and risk factors among young adults in Israel. Br J Ophthalmol, 2017 doi: 10.1136/bjophthalmol-2017-310364.

3. Nelson LB: Macular changes following strabismus surgery confirmed by the use of optical coherence tomography. J Pediatr Ophthalmol Strabismus 53: 10, 2016.

4. Lindstrom M, Tjust AE and Pedrosa Domellof F: Pax7-positive cells/satellite cells in human extraocular muscles. Invest Ophthalmol Vis Sci 56: 6132-6143, 2015.

5. Gong HM, Wang J, Xu J, Zhou ZY, Li JW and Chen SF: Identification of rare paired box 3 variant in strabismus by whole exome sequencing. Int J Ophthalmol 10: 1223-1228, 2017.

6. Altick AL, Feng CY, Schlauch K, Johnson LA and von Bartheld CS Differences in gene expression between strabismic and normal human extraocular muscles. Invest Ophthalmol Vis Sci 53: 5168-5177, 2012.

7. Wang H, Huo X, Yang XR, He J, Cheng L, Wang N, Deng X Jin H, Wang N, Wang C, et al: STAT3-mediated upregulation of IncRNA HOXD-AS1 as a ceRNA facilitates liver cancer metastasis by regulating SOX4. Mol Cancer 16: 136, 2017.

8. Akhade VS, Pal D and Kanduri C: Long noncoding RNA Genome organization and mechanism of action. Adv Exp Med Biol 1008: 47-74, 2017.

9. Aubert G, Strauss KA, Lansdorp PM and Rider NL: Defects in lymphocyte telomere homeostasis contribute to cellular immune phenotype in patients with cartilage-hair hypoplasia. J Allergy Clin Immunol 140: 1120-1129.e1, 2017.

10. Li Y, Xu F, Xiao H and Han F: Long noncoding RNA BDNF-AS inversely regulated BDNF and modulated high-glucose induced apoptosis in human retinal pigment epithelial cells. J Cell Biochem 119: 817-823, 2018.

11. Zhu W, Meng YF, Xing Q, Tao JJ, Lu J and Wu Y: Identification of lncRNAs involved in biological regulation in early age-related macular degeneration. Int J Nanomedicine 12: 7589-7602, 2017.

12. Ye Z, Li Z and He S: Long noncoding RNA associated competing endogenous RNAs are induced by clusterin in retinal pigment epithelial cells. Mol Med Rep 16: 8399-8405, 2017.

13. Dennis G Jr, Sherman BT, Hosack DA, Yang J, Gao W, Lane HC and Lempicki RA: DAVID: Database for annotation, visualization and integrated discovery. Genome Biol 4: P3, 2003.

14. Kanehisa M, Sato Y, Kawashima M, Furumichi M and Tanabe M: KEGG as a reference resource for gene and protein annotation. Nucleic Acids Res 44: D457-D462, 2016.

15. von Mering C, Huynen M, Jaeggi D, Schmidt S, Bork P and Snel B: STRING: A database of predicted functional associations between proteins. Nucleic Acids Res 31: 258-261, 2003.

16. Franz M, Lopes CT, Huck G, Dong Y, Sumer O and Bader GD: Cytoscape.js: A graph theory library for visualisation and analysis. Bioinformatics 32: 309-311, 2016.

17. Langfelder $P$ and Horvath S: WGCNA: An R package for weighted correlation network analysis. BMC Bioinformatics 9: 559, 2008.

18. Ye XC, Pegado V, Patel MS and Wasserman WW: Strabismus genetics across a spectrum of eye misalignment disorders. Clin Genet 86: 103-111, 2014.

19. Lueder GT: Orbital causes of incomitant strabismus. Middle East Afr J Ophthalmol 22: 286-291, 2015.

20. Min X, Fan H, Zhao G and Liu G: Identification of 2 potentially relevant gene mutations involved in strabismus using whole-exome sequencing. Med Sci Monit 23: 1719-1724, 2017.
21. Rajab GZ, Suh SY and Demer JL: Magnetic resonance imaging in dissociated strabismus complex demonstrates generalized hypertrophy of rectus extraocular muscles. J AAPOS 21: 205-209, 2017.

22. Haider AS: Unilateral internuclear ophthalmoplegia, strabismus and transient torsional nystagmus in focal pontine infarction. BMJ Case Rep 2016: bcr2016216503, 2016.

23. Agarwal AB, Feng CY, Altick AL, Quilici DR, Wen D, Johnson LA and von Bartheld CS: Altered protein composition and gene expression in strabismic human extraocular muscles and tendons. Invest Ophthalmol Vis Sci 57: 5576-5585, 2016.

24. Schwalm S, Beyer S, Frey H, Haceni R, Grammatikos G, Thomas D, Geisslinger G, Schaefer L, Huwiler A and Pfeilschifter J: Sphingosine kinase-2 deficiency ameliorates kidney fibrosis by up-regulating Smad7 in a mouse model of unilateral ureteral obstruction. Am J Pathol 187: 2413-2429, 2017.

25. Saika S, Yamanaka O, Okada Y, Tanaka S, Miyamoto T, Sumioka T, Kitano A, Shirai K and Ikeda K: TGF beta in fibroproliferative diseases in the eye. Front Biosci (Schol Ed) 1: 376-390, 2009

26. Choi SU, Kim KW and Moon NJ: Effective treatment for prevention of post-operative adhesion after strabismus surgery in experimental rabbit model: $0.5 \%$ tranilast ophthalmic solution. BMC Ophthalmol 16: 166, 2016.

27. Mahan M and Engel JM: The resurgence of botulinum toxin injection for strabismus in children. Curr Opin Ophthalmol 28: 460-464, 2017.

28. McLoon LK, Christiansen SP, Ghose GM, Das VE and Mustari MJ: Improvement of eye alignment in adult strabismic monkeys by sustained IGF-1 treatment. Invest Ophthalmol Vis Sci 57: 6070-6078, 2016.

29. Levin R, Grinstein S and Canton J: The life cycle of phagosomes: Formation, maturation and resolution. Immunol Rev 273: 156-179, 2016.

30. Steinhauser C, Dallenga T, Tchikov V, Schaible UE, Schutze S and Reiling N: Immunomagnetic isolation of pathogen-containing phagosomes and apoptotic blebs from primary phagocytes. Curr Protoc Immunol 105: 14.36.1-26, 2014.

31. Russell DG: Phagosomes, fatty acids and tuberculosis. Nat Cell Biol 5: 776-778, 2003

32. Jiang M, Esteve-Rudd J, Lopes VS, Diemer T, Lillo C, Rump A and Williams DS: Microtubule motors transport phagosomes in the RPE and lack of KLC1 leads to AMD-like pathogenesis. J Cell Biol 210: 595-611, 2015.

33. Chen YG, Satpathy AT and Chang HY: Gene regulation in the immune system by long noncoding RNAs. Nat Immunol 18: 962-972, 2017.

34. Zheng Y, Liu L and Shukla GC: A comprehensive review of web-based non-coding RNA resources for cancer research. Cancer Lett 407: 1-5, 2017.

35. Jarroux J, Morillon A and Pinskaya M: History, discovery and classification of lncRNAs. Adv Exp Med Biol 1008: 1-46, 2017.

36. Wan P, Su W and Zhuo Y: Precise long non-coding RNA modulation in visual maintenance and impairment. J Med Genet 54: 450-459, 2017.

37. Liu J, Ding $X$, Yuan L and Zhang X: Identification of pterygium-related long non-coding RNAs and expression profiling by microarray analysis. Int J Mol Med 38: 529-536, 2016.

38. Li F, Wen X, Zhang $\mathrm{H}$ and Fan X: Novel insights into the role of long noncoding RNA in ocular diseases. Int J Mol Sci 17: 478, 2016.

39. Gong C, Li Z, Ramanujan K, Clay I, Zhang Y, Lemire-Brachat S and Glass DJ: A long non-coding RNA, LncMyoD, regulates skeletal muscle differentiation by blocking IMP2-mediated mRNA translation. Dev Cell 34: 181-191, 2015.

40. Antunes-Foschini RS, Miyashita D, Bicas HE and McLoon LK: Activated satellite cells in medial rectus muscles of patients with strabismus. Invest Ophthalmol Vis Sci 49: 215-220, 2008.

41. Jin CF, Li Y, Ding XB, Li X, Zhang LL, Liu XF and Guo H: lnc133b, a novel, long non-coding RNA, regulates bovine skeletal muscle satellite cell proliferation and differentiation by mediating miR-133b. Gene 630: 35-43, 2017.

42. Tang R, Zhang G, Wang YC, Mei X and Chen SY: The long non-coding RNA GAS5 regulates transforming growth factor beta (TGF-beta)-induced smooth muscle cell differentiation via RNA Smad-binding elements. J Biol Chem 292: 14270-14278, 2017.

This work is licensed under a Creative Commons Attribution-NonCommercial-NoDerivatives 4.0 International (CC BY-NC-ND 4.0) License. 